\title{
Urgences
}

\section{" Histoire de voir " : historicité et logique narrative dans l'œuvre de Jacques Martin}

\section{Catherine Saouter et Philippe Sohet}

Numéro 32, mai 1991

Lectures de bandes dessinées

URI : https://id.erudit.org/iderudit/025646ar

DOI : https://doi.org/10.7202/025646ar

Aller au sommaire du numéro

Éditeur(s)

Urgences

ISSN

0226-9554 (imprimé)

1927-3924 (numérique)

Découvrir la revue

Citer cet article

Saouter, C. \& Sohet, P. (1991). " Histoire de voir " : historicité et logique narrative dans l'œuvre de Jacques Martin. Urgences, (32), 30-43.

https://doi.org/10.7202/025646ar d'utilisation que vous pouvez consulter en ligne.

https://apropos.erudit.org/fr/usagers/politique-dutilisation/ 


\section{“ Histoire de voir": historicité et logique narrative dans l'œuvre de Jacques Martin Catherine Saouter et Philippe Sohet}

\section{BD et Histoire(s)}

On le sait, l'Histoire fait des histoires. La réalité historique reste une bonne pourvoyeuse de personnages, de contextes, de décors, d'anecdotes pour des auteurs en quête de récits. Dès ses premières apparitions - aux États-Unis comme en Europe la bande dessinée ne manque pas d'y recourir en abondance et l'Histoire demeure une de ses inspiratrices privilégiées. Nous avons déjà, par ailleurs ${ }^{1}$, relevé certains traits typiques de la présence de l'Histoire dans la bande dessinée; qu'il nous suffise de rappeler les innombrables bibliographies de personnages édifiants (Christophe Colomb, Mermoz, Charles de Foucauld... et autres du même sexe), les ouvrages de vulgarisation (Histoire de France en bandes dessinees), la veine épique (de Prince Vaillant à $U_{g a k i}$ ) et le redéploiement éditorial durant les années quatre-vingt des principaux éditeurs français de $\mathrm{BD}$ dans ce créneau (Vécu).

La persistance de cette tradition et la diversité de ses productions permettent également, avec le recul, d'analyser le rapport entre l'Histoire et les histoires, l'Histoire et le récit.

La dynamique entre vérité historique et logique narrative relève d'une délicate interaction car le défi virtuel d'un tel projet n'est rien moins que de réussir à enrichir réciproquement ces deux instances. Une place disproportionnée accordée à l'une des deux conduit inévitablement à deux caricatures du récit historique : le peplum ou le musée en cases.

Longtemps, en effet, les dérivés du récit épique ne s'attachent qu'à la dynamique primaire des rebondissements de l'action, n'accordant à l'Histoire qu'un rôle d'arrière-plan. Le référent historique n'est que l'occasion de sortir de nouveaux artefacts kitschs du coffre d'accessoires (parures, armes,

1 Catherine Saouter et Philippe Sohet, "L'Histoire par la bande ", dans Nuit blanche, $n^{\circ} 22,1986$. 
décors, etc.) afin de multiplier un même narratif, la geste répétitive d'un caractère.

Mais le travers inverse peut également être repéré. Une volonté trop prononcée de valoriser la trame historique et ce qu'elle a à montrer peut conduire à étouffer littéralement la logique narrative par cet impératif d'exposition. Le récit n'est plus que prétexte à l'encyclopédie; les protagonistes se recyclent en guides de musées.

Le véritable enjeu du récit historique est de réussir à créer une symbiose équilibrée. D'une part, la dimension historique doit donner un écho, un relief, une dimension supplémentaire à l'action des protagonistes. Elle doit participer pleinement à la trame narrative trop souvent réduite à des dimensions psychologiques primaires: l'Histoire se fait acteur. D'autre part, la perspective qu'autorise un choix de personnages, leur familiarité et les identifications qu'ils supportent, peut offrir aux lecteurs un support introductif à un autre contexte idéologique, culturel, géographique qui toujours lui échappe, le dépasse: le récit se fait pédagogue.

Reconnaissons-le: certains auteurs parviennent à organiser cette lumineuse alchimie et nous leur savons gré de chefs-d'œuvre comme les séries des Passagers du vent (Bourgeon) ou des Sept vies de l'épervier (Julliard et Cothias) ainsi qu'à des degrés moindres, certains albums de Brandoli et Queirolo (Rebecca) ou de Vink (Le moine fou).

Nous voudrions dans ces quelques pages approfondir certains aspects de cette dynamique entre Histoire et récit en nous appuyant sur la relecture d'une série historique, sans doute la plus connue, l'incontournable saga d'Alix l'intrépide, dessinée et scénarisée par Jacques Martin.

Il s'agira de repérer dans un premier temps l'évolution de ce rapport à l'Histoire tant au niveau des logiques narratives qu'à celui des rhétoriques visuelles et, ensuite, de s'interroger sur les dimensions culturelles et idélogiques qu'elles laissent transparaître.

\section{Jacques Martin: la vitrification de l'Histoire}

Jacques Martin s'est rapidement imposé dans le monde de la bande dessinée par sa prédilection pour les récits historiques. 
32

Un aperçu de ses dernières productions montre à quel découpage de l'Histoire il s'est attaché, puisque, à côté des aventures dans l'antiquité romaine (Alix), lui et ses collaborateurs entretiennent des séries situées au Moyen-Âge (Xan-Jhen), à la Renaissance (Vasco), pendant l'époque napoléonienne (Arno) ou contemporaine (Lefranc). Avec son nouveau héros (Orion), il se propose de parcourir les époques préromaines.

La saga d'Alix qui, en quarante ans, a produit dix-neuf albums (et présidé à la naissance du journal Tintin en 1964), reste incontestablement son œuvre marquante et lui a valu la reconnaissance publique: expositions diverses, traduction en de nombreuses langues - dont le latin -, utilisation à des fins scolaires. Mais, par sa longévité, elle se présente également comme un lieu privilégié pour repérer certains traits typiques de cette dynamique entre Histoire et récit qui nous occupe ici.

Pour ce faire nous nous attacherons particulièrement à trois albums qui nous semblent exemplaires de l'évolution du travail de Jacques Martin: Alix l'intrépide (1948), Le dernier Spartiate (1966) et L'empereur de Chine (1983), respectivement les premier, septième et dix-septième albums de la série.

\subsection{Les logiques narratives}

Aux dires mêmes de l'auteur ${ }^{2}$, Alix l'intrépide a été conçu dans la tradition de la grande Odyssée avec une intrigue qui peut se résumer à une course-poursuite effrénée qui mène Alix de Korsabad, au Proche-Orient, à la Gaule, en passant par Rome. Chemin faisant, cet obscur esclave anonyme se révèle être le fils d'un grand chef gaulois et se voit sous peu confier une mission dont dépend l'avenir de Rome, ni plus ni moins. On aura aisément reconnu ici les ingrédients propres aux feuilletons populaires accumulant les paysages, les rencontres, les coïcidences favorables, à la seule fin d'offrir les situations les plus périlleuses et les rebondissements les plus innombrables. Dans ce récit débridé (trop dense, il envahira une

2 Thierry Groensteen et Jacques Martin, Avec Alix, Bruxelles, Casteman, 1984, p. 87. 
partie de l'album suivant), il n'est guère de temps pour s'attarder à une réelle vraisemblance historique, toute la stratégie narrative étant orientée vers la seule geste de son jeune protagoniste.

Un renversement s'opère dans les productions ultérieures. L'Histoire, avec ses marques, s'introduit davantage et s'immisce en force dans l'espace du récit, refrénant, voire muselant, le rythme narratif.

Ainsi, dans Le dernier Spartiate, Alix est victime de naufrageurs en mer ionienne et se retrouve seul sur une plage alors qu'Enak et le reste de l'équipage sont emmenés en captivité dans un endroit inconnu. En remontant leur piste, Alix parvient à une citadelle secrète, quartier général d'une descendante d'Agamemnon (Adréa), qui fomente le soulèvement des Grecs contre les Romains. Comme il se doit, le récit s'achève sur la délivrance d'Enak et l'écrasement de la citadelle.

$\grave{A}$ bien observer, la trame narrative de cet album est construite autour de deux récits bien distincts: 1) la recherche d'Enak (perdu, retracé, retrouvé, dont il partage la captivité et qu'il aide à s'évader); 2) la lutte contre les ennemis de l'empire (qu'il découvre, observe de l'intérieur; contre qui il organise la révolte des esclaves et qu'il vainc à l'aide des légions).

Le premier récit s'achève sur un échec: ayant presque réussi à s'enfuir, la maladresse d'Enak provoque la capture des deux héros et leur retour en captivité dans la citadelle. Ainsi peut commencer le second récit. La sempiternelle maladresse d'Enak est ici fonctionnelle: elle permet la jonction des deux récits et la liaison entre les deux motifs de la rhétorique spatiale de Martin, la pérégrination et la cité-théâtre.

Dans les récits suivants ces deux motifs se retrouvent de diverses façons mais de manière encore plus évidente dans L'empereur de Chine. Alix et Enak se laissent convaincre par le Grec Mardakios de l'accompagner en Chine où il rejoint son père, précepteur du fils de l'empereur. Sur place, attachés à la personne de ce fils, ils seront témoins et victimes d'intrigues politiques et finiront par obtenir l'autorisation de quitter le pays.

Le programme est ici plus clairement «touristique». Des bouts d'intrigue sont amorcés à travers les deux personnages 
34

grecs, le fils de l'empereur malmené par son père, ou encore à travers le prince Wou Tchi de la cour impériale. Assassinat, mort tragique, nomination honorifique des uns ou des autres se font en coulisse mais sans intervention aucune d'Alix et d'Enak. La préséance est donnée à la pérégrination, qui autorise des descriptions des navires chinois, des vues sur des ports asiatiques, des chemins escarpés avec rencontre de poètes-vagabonds, puis à la cité-théâtre du palais impérial d'été, plantée au milieu d'un lac magnifiquement entouré de montagnes.

Dans cet album, à côté du goût habituel pour l'architecture et le costume, se précise nettement une prédilection accentuée pour les détails ethnographiques (la description minutieuse des rituels funéraires, les arcanes des codes de la justice impériale ou les splendides délires paysagers de la noblesse chinoise).

Ces deux albums permettent de mesurer l'écart dans les stratégies narratives depuis l'épopée d'Alix l'intrépide, mais les motifs récurrents se profilent clairement.

Il faut constater tout d'abord une même gestion des espaces du récit. Dans un premier temps, dans chaque album, l'auteur traite d'une pérégrination, de la mer à l'intérieur des terres en remontant une rivière, des bords occidentaux de l'océan Indien à la mer de Chine. Dans un deuxième temps, il traite de la cité-théâtre: forteresse imaginaire des réfugiés spartiates, palais d'été d'un empereur de Chine. Dans les deux cas, pas d'exposition du retour: dans une case finale, les héros quittent simplement le théâtre des actions.

Le déroulement temporel du récit est lié au temps nécessaire à Alix pour nous faire traverser les espaces du récit, franchir la linéarité de la pérégrination, explorer la circularité de la cité-théâtre et s'en échapper. Cette temporalité est à la mesure de l'univers d'Alix et ne s'ancre pas dans un temps historique qui serait commandé par l'évocation d'un événement dûment daté. Les faits et gestes des protagonistes renvoient à un panorama, non à un fait historique.

Notons aussi une étanchéité croissante entre la dimension historiquement référentielle des protagonistes attachés aux lieux du récit (légion romaine, insurgés spartiates, cour impériale, etc.) et les personnages entourant Alix (Enak, le fils 
d'Adréa, le fils de l'empereur). Depuis le premier album de la série où il se démène en tous sens et prend sur lui le salut de Rome, Alix voit son rôle de plus en plus circonscrit par Jacques Martin. Si, dans Le dernier Spartiate, il est encore activement impliqué - même malgré lui - dans le déroulement des événements qui menacent l'empire, L'empereur de Chine le confine désormais dans un strict rôle de témoin, assistant pour nous aux événements sans jamais en modifier le cours. D'où l'importance, dans ces deux derniers albums, du personnage de jonction qui fait la liaison entre les deux groupes d'acteurs: Galva, ami d'Alix et membre de la légion qui le libère, et Wiong serviteur du fils de l'empereur, qui donne à Alix un soutien indéfectible.

\subsection{Les rhétoriques visuelles}

La récurrence et l'accentuation des rhétoriques visuelles peuvent être illustrées à partir de deux exemples de planches choisies dans ces deux albums.

Le dernier Spartiate, planche 14, avec vue sur la citadelle (figure 1).

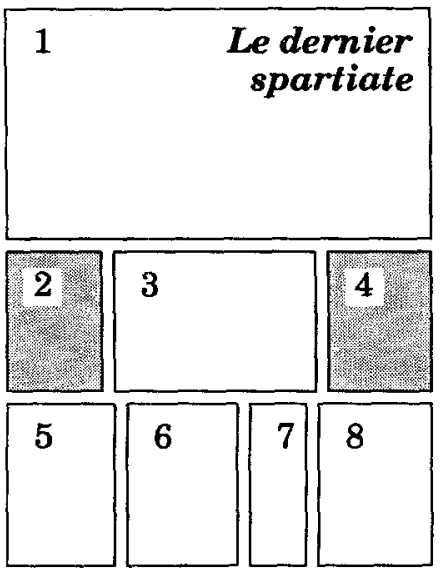

1. plan de grand ensemble légère plongée vue sur la citadelle

2. et 4. plans rapprochés point de vue frontal

Alix regardant $3 / 4$ face et profil 3 et 5. plans d'ensemble vue sur le pont-levis 6 et 7. avant-plan/arrière-plan plongée Alix et ce qu'il voit dos

8. plan rapproché contre-plongée Alix descendant $3 / 4$ face

Très évidents sont, dans cette planche, plusieurs aspects typiques du travail de Martin. Tout d'abord, la vue qui occupe un tiers de la planche. Ligne dhorizon au milieu de la case, 

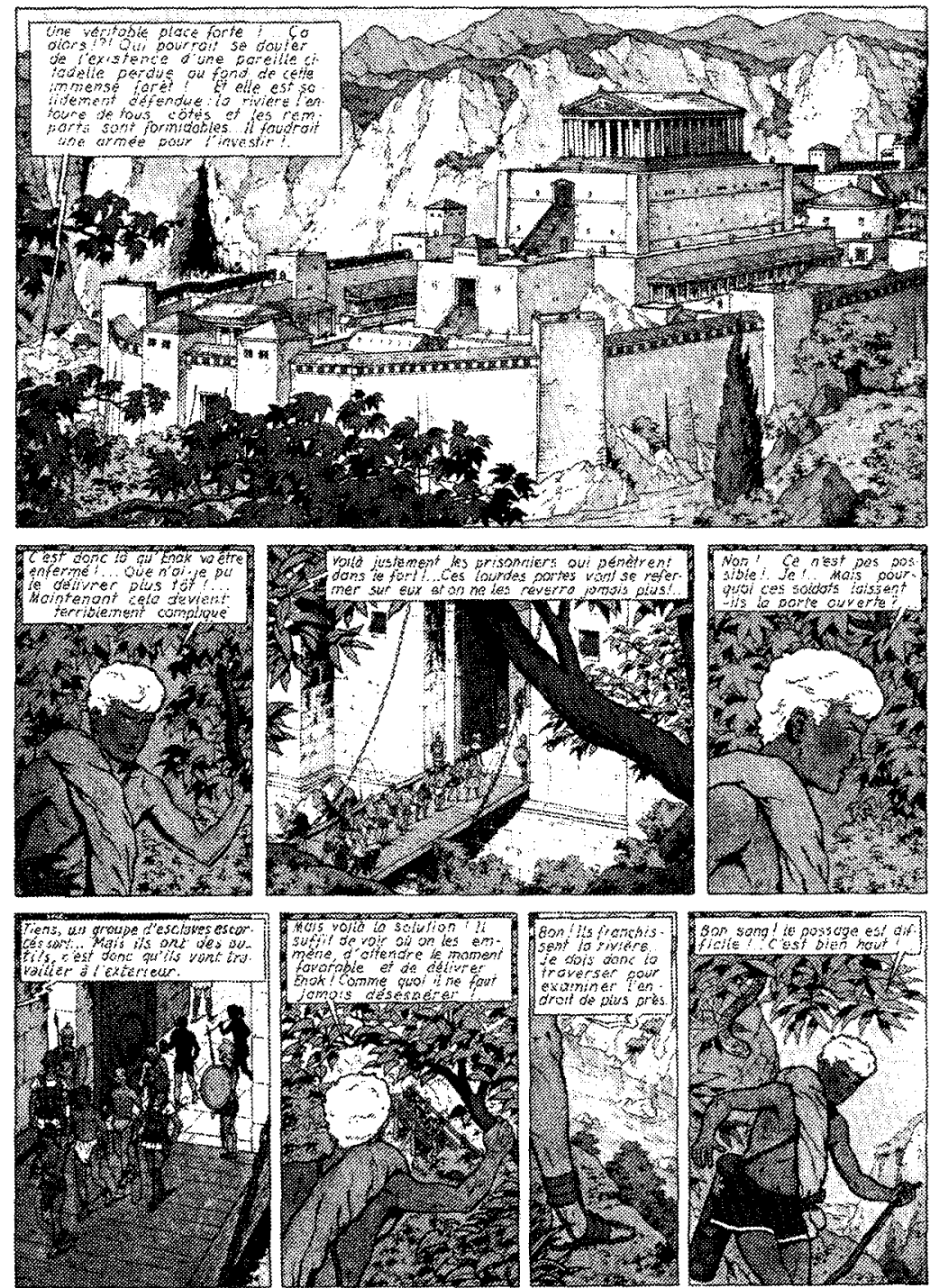

Figure 1 
plan de grand ensemble, point de vue en légère plongée; ces choix concourent à magnifier la citadelle, véritable virtuosité de dessinateur puisqu'on sait qu'il s'agit d'une pure invention - une forgerie - qui eut l'honneur de faire ombrage à une équipe d'archéologues. Cette citadelle est aussi vraie, plus vraie que les vraies, totalement hellénistique.

En deuxième lieu, le goût pour la symétrie dans le bandeau qui contient les cases 2 et 4 . Format et place des cases en question mais aussi, répétition du motif, de la figure du héros. Il s'agit d'une focalisation sur le principal acteur mais aussi et surtout d'une des redondances majeures du dessinateur qui traverse toute sa série: donner à voir non seulement ce qui doit être vu (ici, la citadelle), mais aussi donner à voir celui qui voit. Ailleurs et ad nauseam, Martin dessine le doigt qui désigne ce qui doit être vu. Voyeurisme frénétique qui, ailleurs encore, se signale par des gros plans sur des yeux ou des pupilles regardant depuis des cachettes, par des interstices, des fentes, des oculi.

En troisième lieu, l'indifférence au cinétisme, atout usuel du déroulement narratif de la bande dessinée. Les points de vue sur Alix et ceux sur le pont-levis ne permettent pas d'établir de liaison cohérente entre ce qui est vu et celui qui regarde. Il n'y a pas, par exemple, de construction d'un champ-contrechamp. En fait, les deux bandeaux sont consacrés à l'action suivante: Alix en train de regarder. La première case étant consacrée à une véritable vedute, voilà une planche entièrement consacrée au $v u$ / en train d'être vu / en train de voir. La partie visuelle ne rend compte d'aucune autre forme d'agir et la partie verbale n'y supplée pas, bien au contraire. Alix va quitter son poste d'observation et il justifie son mouvement: «... Je vais donc la traverser (la rivière) pour examiner l'endroit de plus près". Faut-il ajouter que dans la planche précédente, en dernière case, Alix, regardant hors champ, s'exclamait: "Oh!... ça!... c'est fantastique! " Martin prévenait le lecteur qu'il y aurait quelque chose à voir dans la page suivante...

Cette obsession du voir qui subordonne la narration se retrouve entièrement dans une planche similaire de L'empereur de Chine.

L'empereur de Chine, planche 11 , avec vue sur le palais d'été (figure 2). 


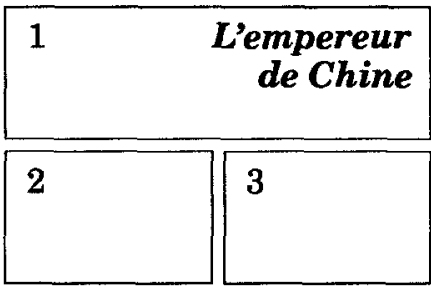

4

Le découpage de cette planche ressemble fort à celui de la précédente. Deux bandeaux précèdent une grande case qui occupe près de la moitié de la planche et qui est consacréee à la vue. Un axe vertical médian passe par l'intercase du deuxième bandeau, se prolonge dans un pilier du pont du premier bandeau et passe par le point central de la grande case, le pavillon principal du palais. Cette fois-ci, point de focalisation sur Alix que l'on distingue à peine, mais ce même détachement envers un enchaînement cohérent des points de vue. Les cases donnent à voir des facettes de ce palais d'été qui ne peuvent en aucune façon être assimilées ni à un déplacement des protagonistes ni à un trajet métaphorique dans le déchiffrement de la planche. D'un point de vue quasi au ras du sol, on passe à une véritable vue aérienne qui n'a d'autre justification que de mieux rendre une vision globale de l'ensemble après la description par facettes entreprise dès la planche précédente. Le dialogue confirme, ici aussi, la prépondérance du voir. "Vous seriez relégués à l'extérieur [...]»/ "[...] Il ne nous resterait plus qu'à retourner »/ "Peut-être! Mais vous auriez fait un beau voyage."/ "Intéressant en tout cas". L'option touristique ne prend même pas prétexte d'Alix pour intégrer à la narration la justification d'un tel déploiement du donné à voir. Alix est un figurant à peine visible, à grande distance du lecteur ${ }^{3}$. Ici également se

3 Dans La Tour de Babel, qui se déroule au Proche-Orient et à Babylone, le récit est carrément interrompu pour une véritable visite guidée au cours de 

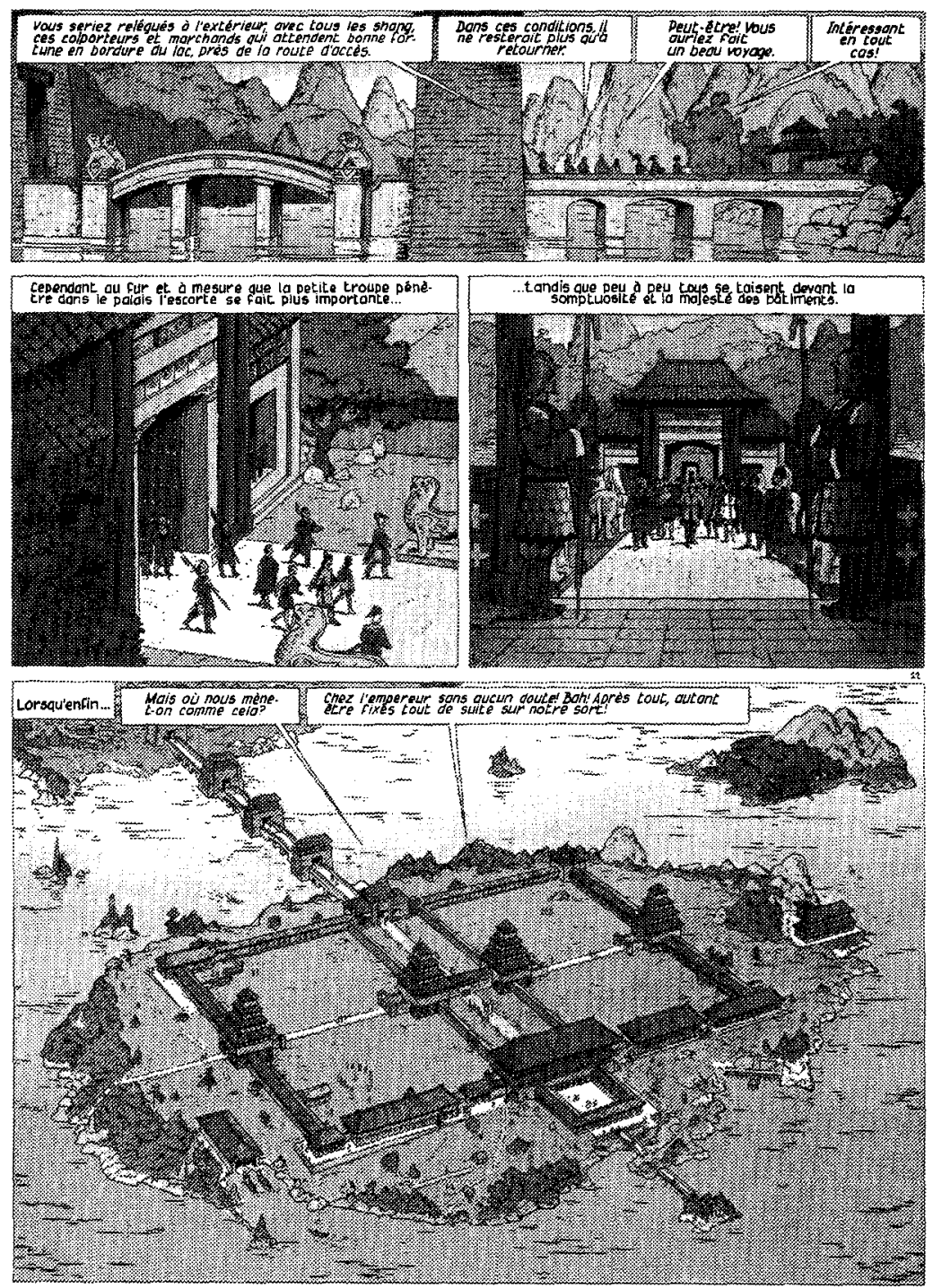
40

retrouve accentué le goût pour la symétrie à travers cet axe médian qui traverse la planche et dans la construction de la troisième case avec son point de fuite central, ses colonnes et ses soldats répartis de part et d'autre de la case.

Dans le domaine de la bande dessinée, de telles stratégies narratives doivent se traduire par des artifices de la rhétorique visuelle. Là encore, du premier album de la série aux derniers, des mécanismes se sont imposés. Alix l'intrépide, avec sa profusion de petites vignettes alignées en bandeaux homogènes 4 , la pauvreté des plans et des enchaînements entièrement soumis à la description de l'action la plus immédiate, intégrait les procédés stylistiques propres au feuilleton. Il ont peu à peu cédé la place à une mise en scène plus propice de la logique d'exposition vers laquelle s'oriente la série.

Ainsi l'écart de dix-sept années qui sépare la réalisation de ces deux albums aura conduit l'auteur à cristalliser et renforcer des logiques narratives et une rhétorique visuelle au service de son projet. Jacques Martin n'est pas un raconteur d'histoire, il est un montreur de vues.

\section{Pompiérisme et BD}

Que ce soit par les thèmes historiques, par les choix narratifs ou par les stratégies visuelles, le travail de Martin renvoie à une tradition des Beaux-Arts bien connue: la dégénérescence du néo-classicisme en pompiérisme.

La connaissance de l'Antiquité romaine de Martin semble passer par la vision qu'en ont donnée David et ses émules, jusqu'à Bouguereau et autres Boulanger, et son travail formel est marqué par les tics propres à cette école: hiératisme des postures, dominance de la composition symétrique, simulacre de la reconstitution.

laquelle Alix trouve très amusant d'aller manger dans les souks. Ce thème de la visite guidée, qui couvre encore les premières planches du Dieu sauvage, peut passer de la dimension géographique à la dimension historique par des artifices narratifs: le voyage à Babylone est le prétexte à décrire l'époque (révolue) qui aurait vu s'ériger cette architecture. Quant au Cheval de Troie, il ne nous propose rien d'autre qu'un remake de l'épisode célèbre antérieur de plus d'un millénaire. 4 Pas loin de seize cases par planche en moyenne, alors que ce ratio diminuera régulièrement pour se fixer à huit dans L'empereur de Chine. 
L'Antiquité peinte par ces peintres n'est pas cette période complexe, longue de plusieurs millénaires, ponctuée d'une diversité de civilisations dont l'héritage est plus ou moins clair dans nos mémoires collectives. L'Antiquité est romaine, indifféremment républicaine ou impériale, surtout pas tribale, ni en voie de christianisation. Dépolitisée, elle est le creuset de la magnificence de valeurs héroïques et viriles, éternellement figées dans des tableaux altiers dans lesquels priment la symétrie, l'atemporalité, la verticalité. Apologies du corps, de l'architecture et de l'artefact, les tableaux néoclassiques et surtout pompiers sont la trace d'un idéal de puissance accompli sans contingences socio-historiques ni diachronies lourdes d'errements politiques.

En bonne logique, nous retrouvons chez Martin les options idéologiques qui soutenaient déjà le travail des académiques. En effet, cette méticulosité de reconstruction dans les artefacts qui fit la réputation de Martin cohabite quelquefois avec des "relâchements" étonnants. Dans Le dernier Spartiate, Martin invente de toutes pièces des vélléités d'insoumission à l'ordre romain qui auraient animé les Grecs. Dans L'empereur de Chine, il s'inspire d'un Romain qui aurait fait la même navigation et évacue les cohortes de marchands anonymes qui parcouraient l'océan Indien. En évitant de traiter de trop près l'histoire événementielle, il intègre des faits qui renseignent davantage sur son approche idéologique que sur son bagage de connaissances.

Peu importe qu'il falsifie l'attitude des Grecs romanisés nous sommes ici dans l'ordre de la fiction - , ce qui compte est qu'il choisisse le thème de la volonté de puissance, de l'hégémonie impérialiste. Ce thème est récurrent dans la série et nous pourrions le circonscrire dans chacun des albums. Vercingétorix, Iorix, Arbacès, Sardon, Oribal, Kildéric, etc, sont autant de clones d'Adréa réitérés tout au long de la saga.

L'option fondamentale du travail de Martin reste conforme : évacuer les dimensions collectives, faire silence sur les tissus sociaux et culturels, valoriser les figures héroïques et les individus passés à l'histoire. La Chine est donnée à voir à travers un empereur, son système féodal à travers un prince, la classe des voyageurs est évoquée par un précepteur, corps de métier plutôt éloigné du corps des marchands. L'Histoire, telle que Martin l'envisage, est une succession de prises et de 
pertes du pouvoir, dont il retient les héros heureux ou malheureux, tous chefs militaires ou politiques. Les aventures d'Alix ne mettent en scène que la classe des patriciens. Là sont choisis les acteurs des péripéties, dont l'ultime est César, tous les autres étant d'un ordre second, quasi décoratif, à l'exception de ces personnages, tous plébéiens, secondaires au plan historique, mais primordiaux au plan des stratégies narratives - les passeurs - qui assurent la jonction entre la famille de papier et les acteurs historicisants.

On pourrait juger falsifiantes de telles options, elles reflètent plutôt une certaine vision de l'Histoire qui n'est bien évidemment pas singulière au seul Martin et dont l'analyse n'est pas réellement l'objet de ces notes. Par contre il était opportun de souligner les impacts restrictifs d'une telle façon de voir sur les stratégies narratives et les rhétoriques visuelles.

Martin confirme en effet l'uniformité de sa démarche en construisant des narrations qui évacuent l'agir, donc les possibilités d'interférence et d'interaction. L'indifférence croissante de son héros est fonctionnelle. Elle protège le système d'une mise en contradiction. D'où l'utilité de ces passeurs entre la trame historique et les personnages de la série. Un personnage et un seul assure la cohabitation vraisemblable de deux groupes disjonctifs.

L'approche visuelle de Martin est, elle aussi, tout à fait cohérente. Il procède par exposition des artefacts, bâtiments, armes, objet, costumes et il les donne à voir exposés dans des cases avec un grand souci de lisibilité. La logique d'exposition l'emporte sur la logique de contextualisation puisque l'Histoire n'est pas un milieu mais une collection. La planche est donc un lieu de fractionnement pour disposer des vues et non un découpage dont les éléments sont interdépendants.

En détournant, au plan de la dynamique entre Histoire et récit, la formule classique de Fresnault-Deruelle, la série de Martin serait passée, d'Alix l'intrépide à ses dernières parutions, d'un récit linéaire à un exposé tabulaire et aurait donné ainsi, dans le champ de la bande dessinée, un héritier surprenant à un mouvement pictural mort au tournant de ce siècle, en totale et incongrue hétérogénéité avec la modernité.

Avec de tels dispositifs, nous sommes loin des frénésies narratives des premières aventures d'Alix. L'Histoire s'est 
glissée dans les cases par le biais du décor et de ses détails, a vampirisé peu à peu toute la dynamique narrative jusqu'à imposer son propre et seul rythme: celui de la visite guidée. 